\title{
Monitoring of Valve Gap in Diesel Engine Based on Vibration Response Feature Extraction
}

\author{
Chaoming Huang, ${ }^{1,3}$ Jie Li, ${ }^{1}$ Xin Wang, ${ }^{2}$ Jianbin Liao, ${ }^{3}$ \\ Hongliang Yu, ${ }^{3}$ Chih-Cheng Chen, ${ }^{4,5}$ and Kun-Ching Wang ${ }^{6 *}$ \\ ${ }^{1}$ College of Maritime Engineering, Dalian Maritime University, Dalian Liaoning 116026, China \\ ${ }^{2}$ Information and Communication Office, China Coast Guard 100061, China \\ ${ }^{3}$ College of Marine Engineering, Jimei University, Fujian 361021, China \\ ${ }^{4}$ Department of Automatic Control Engineering, Feng Chia University, Taichung 40724, Taiwan \\ ${ }^{5}$ Department of Aeronautical Engineering, Chaoyang University of Technology, Taichung 413310, Taiwan \\ ${ }^{6}$ Department of Mechanical and Computer-Aided Engineering, Taichung 40724, Taiwan, R.O.C.
}

(Received December 31, 2020; accepted March 24, 2021; online published April 16, 2021)

Keywords: diesel engine valve clearance, vibration feature extraction, time-frequency image, image segmentation

To evaluate the working state of a diesel valve by vibration response signal analysis and carry out fault diagnosis, a method is proposed to resolve the problem of how to effectively extract the vibrational characteristic parameters of the valve seat from a nonstationary vibration signal on the surface of a diesel engine by using local wave decomposition and reconstruction technology, continuous wavelet transform (CWT) time spectrum images, and image processing technology. The difference in the energy distribution characteristics of the vibration signal time and frequency domains of the diesel engine is enhanced by the local wave decompositionreconstruction analysis method, the time spectrum map of the reconstructed signal is drawn by using the CWT, the vibration characteristics and corresponding features are extracted by image segmentation technology, and the intrinsic relationship between the gas valve gap and the gas valve landing shock vibration characteristics is discussed. Gas valve clearance status identification and the diagnostic basis function are established, and the quantitative monitoring of the air valve clearance of the diesel engine and the fault diagnosis of abnormal gas valve clearance are realized.

\section{Introduction}

Valve/valve-seat insert pairs are important moving pairs of the engine cylinder head and are affected by the high-temperature and high-pressure gas. When closed, the valve collides with and wears the valve seat, which generates dry friction between the valve surface and the seat surface, thus aggravating the wear of the valve. ${ }^{(1,2)}$ The valve gap changes because of wear, decreasing the scavenging efficiency, fuel filling coefficient, thermal efficiency, and power output, which is accompanied by increased toxic exhaust gas emissions and corrosion damage to

*Corresponding author: e-mail: kunching.wang@gmail.com https://doi.org/10.18494/SAM.2021.3289 
mechanical components. ${ }^{(3,4)}$ The conventional methods to monitor and regulate the running state of a valve gap are feeler gauge measurement and the use of an auto-compensating device. Feeler gauge periodic measurements and adjustments are performed manually with the engine shut down and disassembled at preset maintenance intervals, regardless of the engine's current state. The engine should be in a cold state during the measurement, and the ambient temperature should not be too high to ensure the reliability of the measurement results. The autocompensating method inserts an extra mass in the timing gear or installs a hydraulic adjustment device, increasing the possibility of damage to the timing system, leading to an uncontrolled increase in the valve clearance and structural failure. ${ }^{(5)}$ Moreover, compensation devices cannot be installed on some occasions in the case of compact structure requirements.

Thus, a new method is required to monitor and diagnose valve-seat health by real-time analysis. Many attempts to develop such a method have been carried out using vibration sensors. Vibrations of the engine cylinder head caused by imbalance, wear, and looseness are accompanied by violent shock vibration of the valve, which is transmitted to the diesel engine surface through the gas valve mechanism. Vibration sensors detect the frequency, amplitude, and impact of vibrations on the diesel engine surface. These parameters provide valuable information to diagnose faults and to monitor the condition of the valve seat. The data from the sensors are processed to extract and study the information on the working state of the gas valve and seat to obtain these parameters. This process needs the law of vibration to establish the intrinsic relationship between the data and the parameters.

Many researchers have studied this topic and put forward a series of methods such as time spectrum analysis, ${ }^{(6,7)}$ wavelet packet analysis, ${ }^{(8-10)}$ independent component analysis (ICA), ${ }^{(11,12)}$ variational mode decomposition, ${ }^{(13-15)}$ singular value decomposition, ${ }^{(16,17)}$ local normal decomposition, empirical modal decomposition, and the use of neural networks or fuzzy clusters. ${ }^{(18,19)}$ It is usually difficult for time spectral maps to achieve on-line monitoring. The wavelet type influences the wavelet analysis method, and inappropriate wavelet types will cause large errors in the diagnosis results. When analyzing nonlinear and unstable vibration signals, local normal decomposition and empirical modal decomposition have disadvantages, such as endpoint effects and ambiguous modality, which have adverse effects on state monitoring and troubleshooting.

To improve the accuracy of nonlinear and nonsmooth vibration signal decomposition on a diesel engine surface, as well as the accuracy of vibration feature extraction and status recognition, we propose the use of local wave decomposition, reconstruction technology, timefrequency images during the continuous wavelet transform (CWT), and image processing technology to extract the characteristic parameters of the valve-seat vibration signal. The local wave decomposition-reconstruction method is used to process the surface vibration signal of diesel engines, and the characteristic energy parameters in the time and frequency domains are extracted by using the CWT and image segmentation technology. The spatial and texture feature parameters of the secondary image are extracted, and a diagnostic criterion function based on the characteristic parameters is established to quantitatively monitor and diagnose the valve clearance of diesel engines. A test was carried out on a WD615 diesel engine bench to study the effectiveness of the proposed method of diesel engine valve clearance fault diagnosis. 


\section{Local Wave Decomposition Algorithm for Nonstationary Time-varying Signal Analysis}

Owing to the system's nonlinearity and the external environment, the vibration signal of a diesel engine is usually nonstationary, so the analysis of nonstationary signals is a prerequisite for the correct analysis of the diesel engine vibration signal. The main characteristic of a nonstationary nonlinear signal is its time-varying transient frequency, which only exists in a certain local time, and this kind of signal is called a local wave. ${ }^{(20,21)}$

Decomposing nonlinear nonstationary signals into a finite number of basic mode components with instantaneous frequency characteristics is called local wave decomposition, also known as the screening process. A stopping criterion for the screening process can be realized by limiting the standard deviation between two successive processing results, given by

$$
S_{d}=\sum_{t=0}^{T} \frac{\left|h_{1(k-1)}(t)-h_{1 k}(t)\right|^{2}}{h_{1 k}^{2}(t)} .
$$

Typical values of $S_{d}$ range from 0.2 to 0.3 . In accordance with the restriction conditions of the basic mode component, the original signal $X(t)$ is processed $k$ times to obtain the first basic mode component $c_{1}$, where

$$
c_{1}=h_{1(k-1)}-m_{1 k} \text {. }
$$

After $c_{1}$ is obtained, the difference between $X(t)$ and $c_{1}$ is used as the signal to be sifted repetitively, and then the second basic mode component can be obtained. By analogy, $n$ basic mode components can be obtained until the process meets the preset stop conditions, then the process can be stopped. Finally, we acquire the following results:

$$
\begin{aligned}
X(t) & =\sum_{j=1}^{n}\left\{h_{j\left(k_{j}-1\right)}-m_{j k_{j}}\right\}+r_{n} \\
& =\sum_{j=1}^{n} c_{j}+r_{n} .
\end{aligned}
$$

The stop condition can be taken as follows: (1) stopping occurs when the last component $c_{n}$ or the remaining component becomes smaller than the predetermined value; (2) when the residual component becomes a monotonic function, the basic mode component cannot be filtered out. The residual component is either an average trend or a constant.

The Hilbert transform is carried out for each component $c_{j}(t)$, and the instantaneous frequency is calculated using $\omega(t)=d \theta / d t$, then $X(t)$ can be expressed as follows: 


$$
X(t)=\operatorname{Re} \sum_{j=1}^{n} a(t) e^{i \int \omega_{j}(t) d t} .
$$

It can be seen that each basic mode component can be amplitude- or frequency-modulated. A variable amplitude and instantaneous frequency improve the signal decomposition efficiency and make this decomposition method more capable of analyzing nonlinear and nonstationary data.

\section{Experimental Design}

\subsection{Test bench}

A test was carried out on a WD615 diesel engine bench controlled by an FC2000 engine measurement and control system. Different operating conditions (e.g., speed, load) can be set according to the test requirements to study the diesel engine's performance and the state of parts and contact pairs under different operating characteristics such as load and speed. The WD615 diesel engine bench is shown in Fig. 1. The normal values of the diesel engine valve's cold clearance are $0.3 \mathrm{~mm}$ for the intake valve and $0.4 \mathrm{~mm}$ for the exhaust valve.

\subsection{Design of test scheme for abnormal valve clearance}

The valve failure test of the WD615 diesel engine was carried out on the bench, and the wear fault identification and diagnosis methods of the valve were studied. The diesel engine operates under four different valve failure conditions, and the working conditions are shown in Table 1. All the clearance values in the table are valve clearance values when the diesel engine is cold. Therefore, it is necessary to wait at least one day after each test before changing the working condition and adjusting the clearance. A YJ9A7018 piezoelectric acceleration sensor with a sensitivity of $4.52 \mathrm{pC} / \mathrm{ms}^{-2}$ and a range of $\pm 500 \mathrm{~g}$ was used to measure the vibration signal on the surface of the head of cylinder 1. The installation test of the sensor is shown in Fig. 2. The sampling frequency is $4096 \mathrm{~Hz}$ and the number of data samples is 1024 points. After the DLF-8 charge voltage filtered the integral amplifier, the cylinder head vibration signal with the

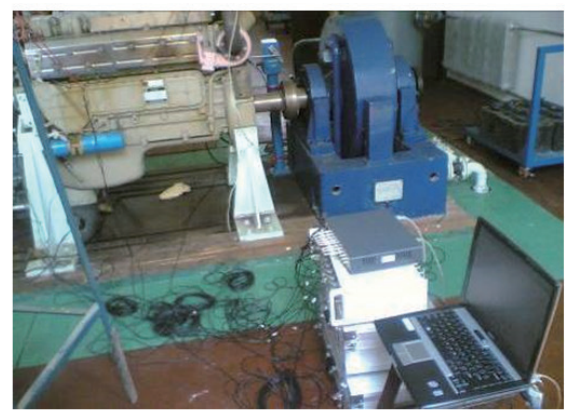

(a)

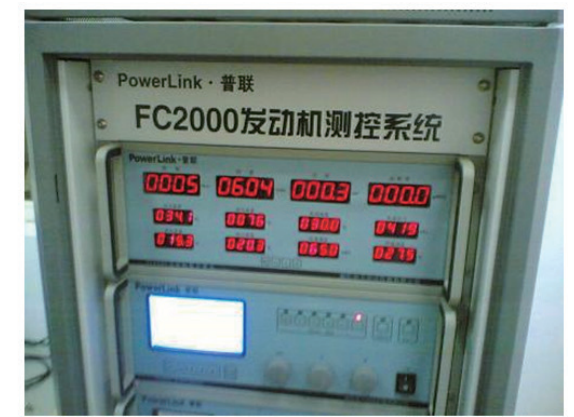

(b)

Fig. 1. (Color online) WD615 diesel engine bench. (a) Test bench and signal test system. (b) Control system. 
Table 1

Test conditions of the diesel engine.

\begin{tabular}{lccccc}
\hline Number & Working condition & $\begin{array}{c}\text { Exhaust valve } \\
\text { clearance }(\mathrm{mm})\end{array}$ & $\begin{array}{c}\text { Inlet valve } \\
\text { clearance }(\mathrm{mm})\end{array}$ & $\begin{array}{c}\text { Rotation speed } \\
(\mathrm{rpm})\end{array}$ & $\begin{array}{c}\text { Torque } \\
(\mathrm{N} \cdot \mathrm{m})\end{array}$ \\
\hline Val1 & Exhaust valve clearance is too small & 0.3 & 0.3 & 1800 & 500 \\
\hline Val2 & Exhaust valve clearance is small & 0.35 & 0.3 & 1800 & 500 \\
\hline Val3 & Normal clearance & 0.4 & 0.3 & 1800 & 500 \\
\hline Val4 & Exhaust valve is too large & 0.45 & 0.3 & 1800 & 500 \\
\hline
\end{tabular}

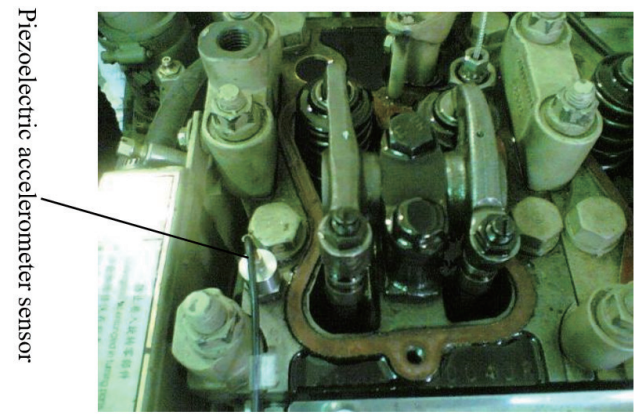

Fig. 2. (Color online) Layout of diesel engine vibration measuring points.

sampling frequency was extracted using an INV306V intelligent signal acquisition and processing analyzer of Dongfang Institute and a DASP2006 professional intelligent data acquisition and signal analysis system. The vibration signals of the diesel engine under different valve clearances were collected. Before the signal test of each working condition, the diesel engine was run for about $45 \mathrm{~min}$, and we started to collect data once the diesel engine was working stably.

\section{Experimental Results and Analysis}

In accordance with the above test scheme, the test was carried out on the WD615 diesel engine test bench. The clearance between the rocker arm and the diesel engine's valve rod was adjusted to simulate the wear of the moving pair of the diesel engine's valve mechanism, which resulted in a change in the matching and abnormal clearance. By considering the vibration response, the method of gas valve fault diagnosis was studied.

\subsection{Power spectrum analysis and local wave processing}

Results for Val1, Val2, Val3, and Val4 were collected (corresponding to valve clearances of $0.3,0.35,0.4$, and $0.45 \mathrm{~mm}$ when the diesel engine is cold, respectively). Denoising and reconstruction based on the above-mentioned local wave decomposition were carried out, and the power spectrum signals were calculated. The results are shown in Figs. 3-6. 


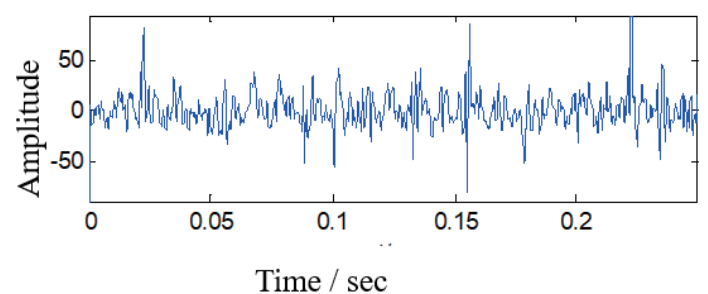

(a)

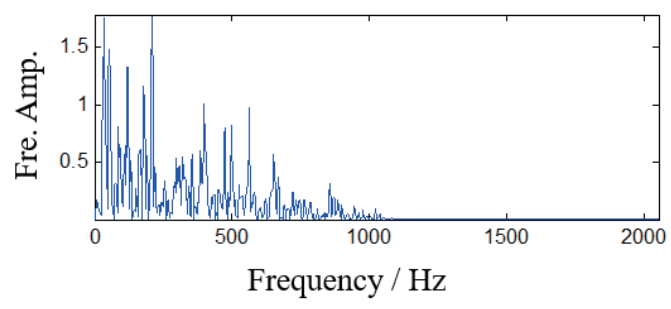

(b)

Fig. 3. (Color online) Processing results of vibration signal in test Vall. (a) Local wave processing result. (b) Power spectrum of the result signal.

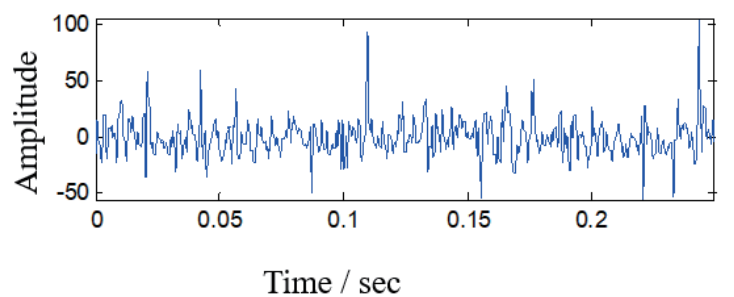

(a)

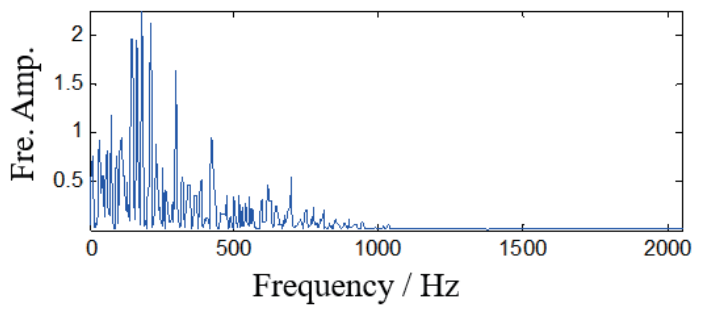

(b)

Fig. 4. (Color online) Processing results of vibration signal in test Val2. (a) Local wave processing result. (b) Power spectrum of the result signal.

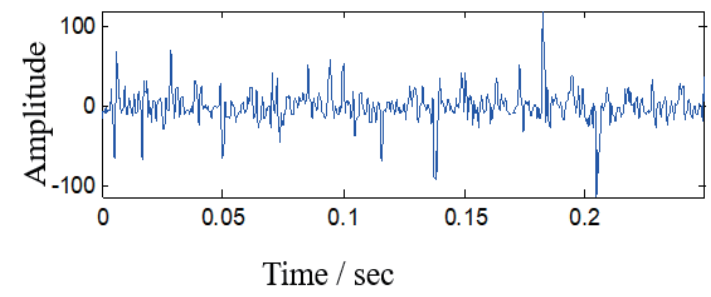

(a)

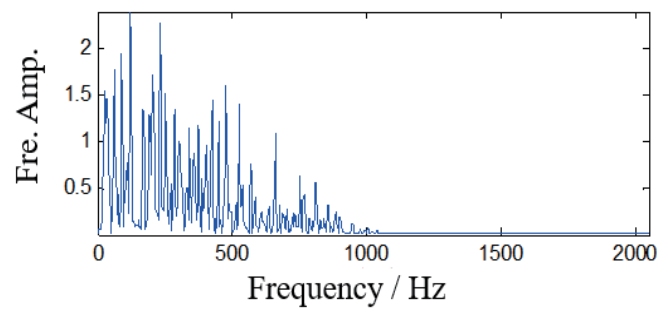

(b)

Fig. 5. (Color online) Processing results of vibration signal in test Val3. (a) Local wave processing result. (b) Power spectrum of the result signal.

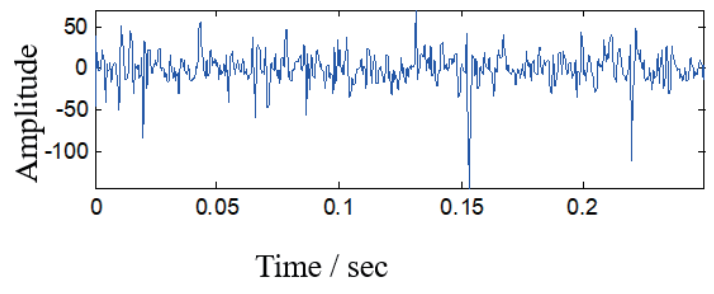

(a)

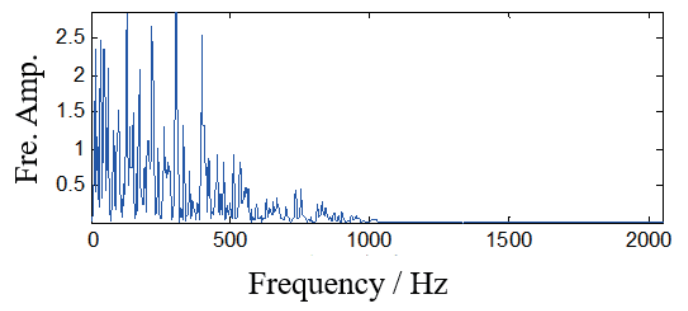

(b)

Fig. 6. (Color online) Processing results of vibration signal in test Val4. (a) Local wave processing result. (b) Power spectrum of the result signal.

According to the time-domain waveform of the local wave processing results, the absolute value of the amplitude of the time-domain vibration signal monotonically increased with the sequential change in the working conditions from Vall to Val4, reflecting the increase in vibration energy and vibration severity. In the power spectrum signal waveform, it can be seen that the main energy of each working condition was concentrated between 0 and $500 \mathrm{~Hz}$, and a 
vibration energy peak appears near $200 \mathrm{~Hz}$. With the change in working conditions from Vall to Val4, the energy peak of the power spectrum signal increased gradually, and the vibration energy peak near $200 \mathrm{~Hz}$ became more significant. The frequency bandwidth of the vibration energy peak area gradually widened, which indicates that the overall energy of the vibration increased along with the complexity. The above time-domain and power spectrum signals are closely related to the diesel engine valve clearance under various working conditions. According to the test design, the set value of valve clearance gradually increased under these conditions. The clearance of the valve affects the opening and closing times of the valve and the velocity and acceleration of the valve when it opens and closes. Thus, such clearance affects the impact force and energy of the valve seat. With increasing clearance, the energy of the impact vibration and the intensity of the impact vibration increased. This is consistent with the results of signal analysis. Therefore, using the energy characteristics and distribution characteristics of timedomain signals and power spectrum signals after local wave decomposition, noise reduction and reconstruction can be extracted to describe the signal characteristics. The working state of the valve can then be characterized.

\subsection{CWT secondary image analysis}

After denoising and reconstruction based on local wave decomposition, a Morlet wavelet with good time-frequency local performance was used for the CWT to process vibration signals; continuous wavelet decomposition can analyze the signals in different frequency intervals of the original signal by scaling to identify different vibration characteristics.

The three-dimensional curved surface spectrum and isogram of the secondary image formed by the CWT energy time spectrum can directly describe the signal's time-frequency characteristics and reflect the state of the signal on the time-frequency phase plane. The CWT equivalent time spectrum and three-dimensional time spectrum under the four different working conditions of Vall to Val4 were calculated, and the results are shown in Fig. 7.

From the CWT secondary image, it was observed that the vibration signal energy of the diesel engine cylinder head was mainly concentrated in the range of $0-500 \mathrm{~Hz}$, which is consistent with the above time history curve and the results of power spectrum signal characteristic analysis. However, the distribution form is different. Under working condition Val1, the main energy of the vibration signal in the frequency distribution was relatively sparse and scattered. From Vall to Val4, the frequency distribution of the spectrum energy is concentrated in the low-frequency band and the area of vibration with a larger energy increases. The figure shows that the energy distribution of the low-frequency position of the timefrequency spectrum of working condition Vall was not significant, and the frequency spectrum corresponding to working conditions Vall to Val4 appeared periodically from 0 to $300 \mathrm{~Hz}$ in the frequency domain, and the low-frequency ripple band became more obvious, resulting in a larger energy distribution area (around 200-300 Hz). It can also be seen that the forms of the spectrum distribution (shape and position of the three-dimensional spectrum and equivalent spectrum) during the CWT showed obvious differences and a regular distribution under different valve clearance conditions. Different distribution laws correspond to different clearance conditions. 

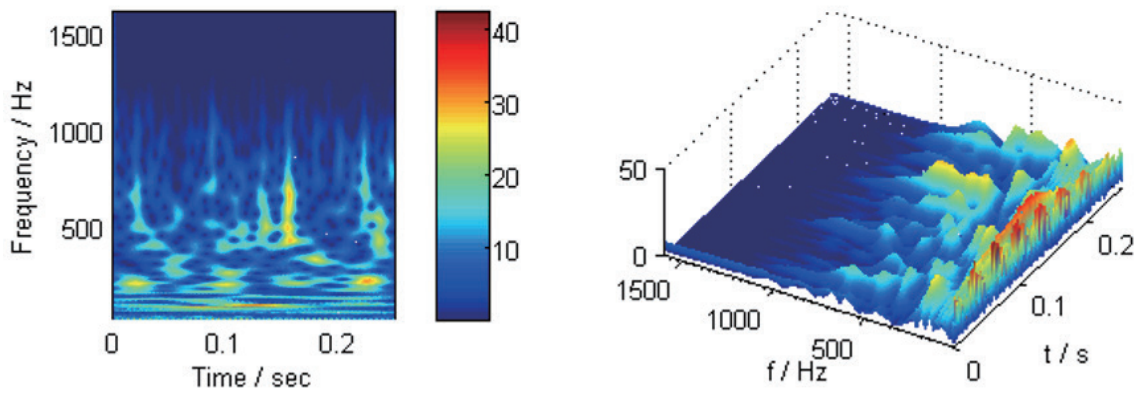

(a)
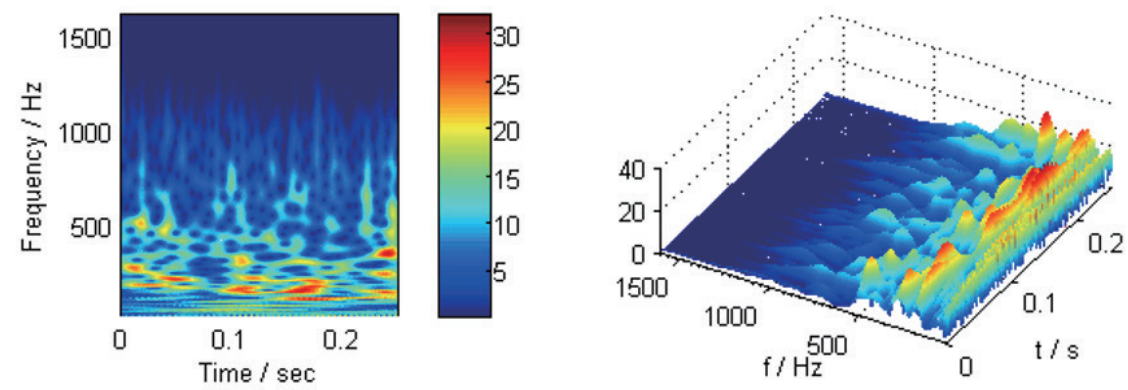

(b)
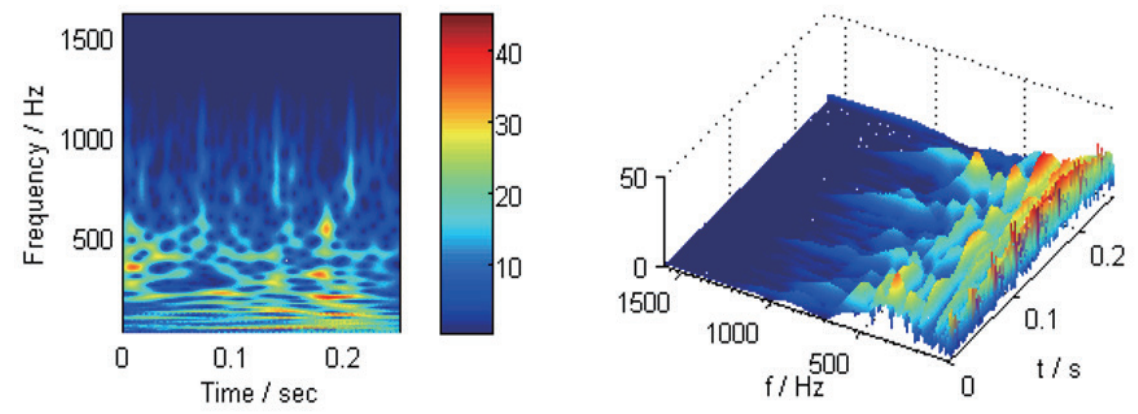

(c)
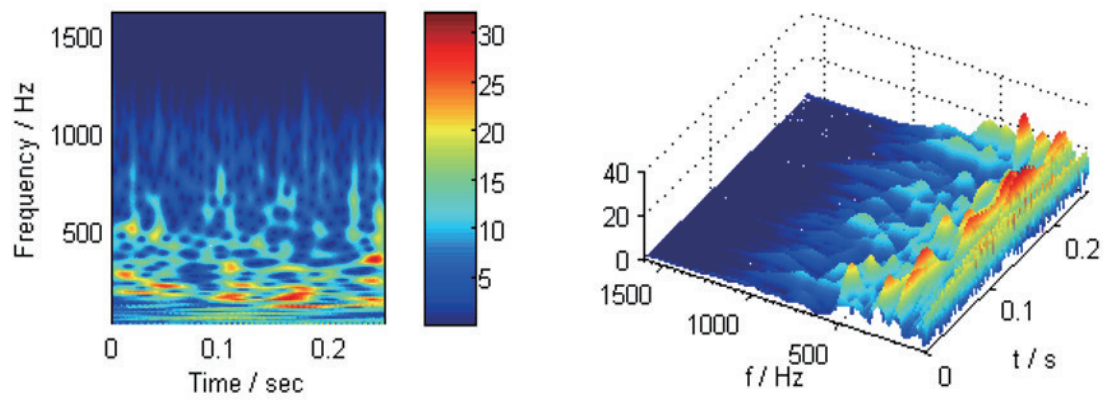

(d)

Fig. 7. (Color online) CWT equivalent time-frequency spectrum and 3D time-frequency spectrum. (a) Test Vall, (b) Test Val2, (c) Test Val3, and (d) Test Val4. 
Therefore, quantitative characterization and pattern recognition of the valve clearance state can be carried out by extracting the distribution characteristic parameters of the CWT spectrum.

\subsection{Characteristic parameter extraction}

For quantitative analysis, the time-domain maximum peak value $A_{m t}$, the time-domain second moment $E_{t}$, the frequency-domain maximum peak value $P S D_{m}$, and the frequencydomain total energy $E_{f}$ were selected as characteristic parameters of the time history curve and the power spectrum of the vibration signal processed by the local wave denoising and reconstruction. These characteristic parameters are described as follows.

Maximum peak value $\left(A_{m t}\right)$

$A_{m t}$ is the maximum peak value of the vibration signal in the time domain (i.e., total vibration level) and reflects the energy of the main impact.

Second moment $\left(E_{t}\right)$

The second moment in the time domain $E_{t}$, which reflects the total vibration energy, is obtained from the following formula:

$$
\begin{aligned}
& \quad E_{t}=\sum_{r=0}^{N-1} x^{2}(r / \Delta t) \cdot \Delta t \\
& N \text { - number of sampling points; } \\
& r \text { - time series } r=0,1,2, \ldots, N-1 ; \\
& \Delta t \text { - sampling interval. }
\end{aligned}
$$

Power spectrum peak value $\left(P S D_{m}\right)$

The power spectrum maximum peak $P S D_{m}$ in the frequency domain reflects the impact energy at a certain characteristic frequency.

Total energy $\left(E_{f}\right)$

The frequency-domain total energy $E_{f}$ is given by

$$
E_{f}=\sum_{i=0}^{N-1} P S D(i) \cdot \Delta f
$$

$P S D(i)$ - power spectral value corresponding to the $i$ th spectral line;

$N$ - number of spectral lines in the power spectrum;

$\Delta f$ - frequency resolution.

In addition, the Otsu threshold method was used to segment the CWT secondary image of the vibration signal, and the results are shown in Figs. 8(a)-8(d) (the left image is the binary result of image segmentation, and the right image is the extracted characteristic object of the vibration signal). 

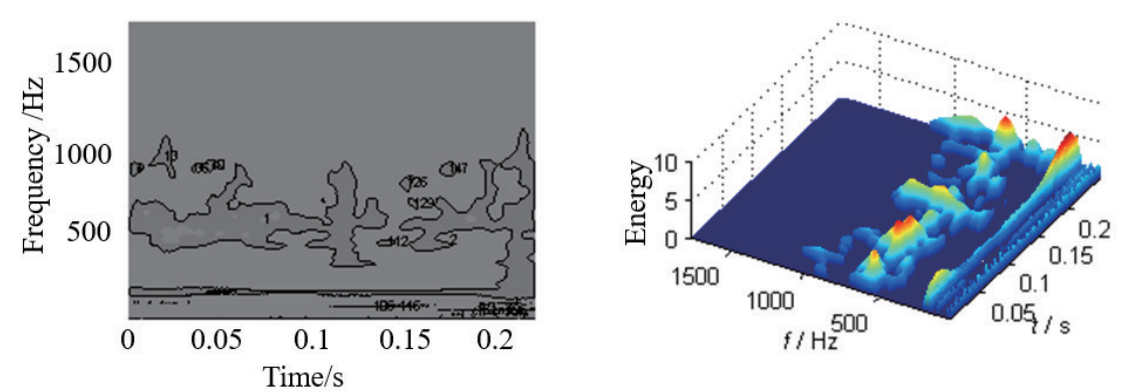

(a)
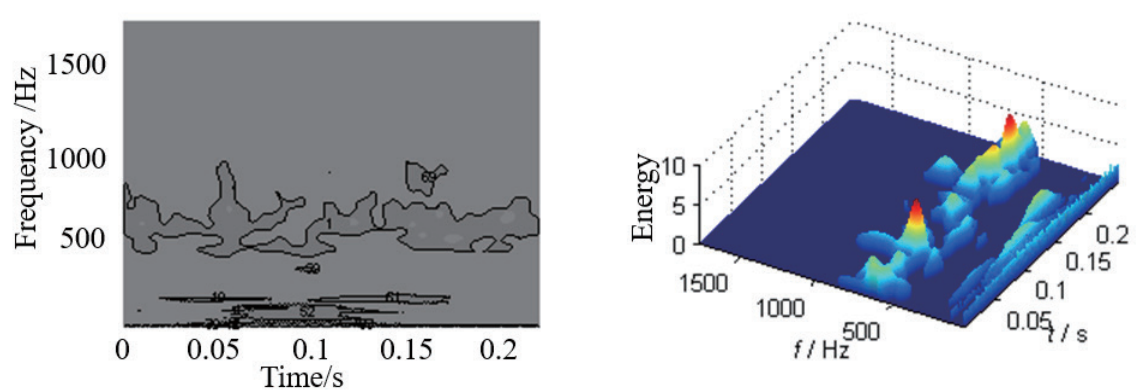

(b)
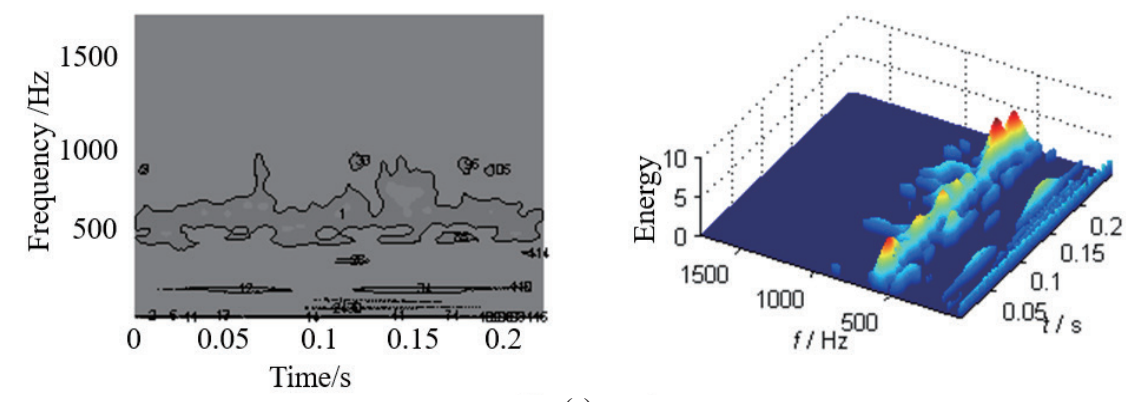

(c)
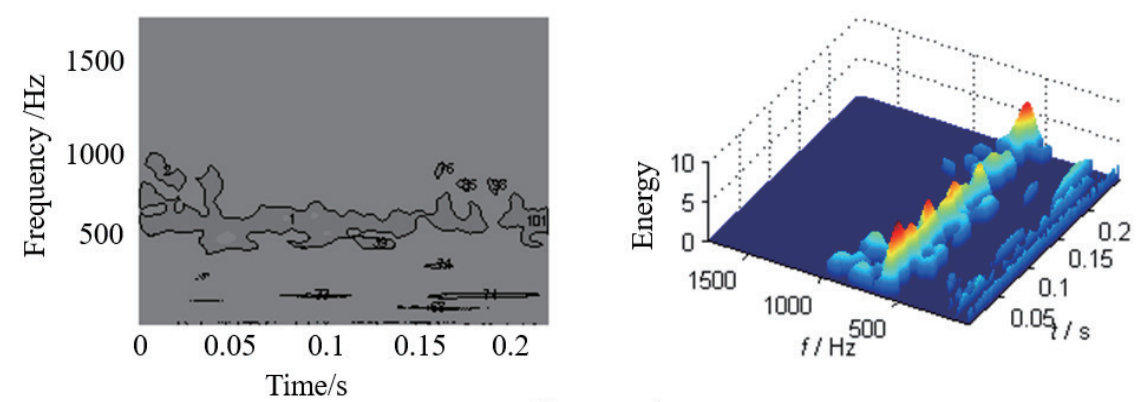

(d)

Fig. 8. (Color online) Segmentation of CWT TFI image. (a) Test Val1, (b) Test Val2, (c) Test Val3, and (d) Test Val4. 
It can be seen that the secondary image segmentation extracted the typical and prominent information dominated by the energy in the time-frequency image, removed the background noise, and retained the useful information in the original image to the maximum extent. Also, from the extracted characteristic objects (the segmented objects of the vibration signals) under the four working conditions, it was found that the shape and spatial characteristics of the characteristic objects showed differences under different working conditions. Therefore, the vibration signals under the four working conditions can be quantitatively analyzed by extracting these features as follows.

Average gradient of segmented object $\left(G_{\text {mean }}\right)$

The average gradient $G_{\text {mean }}$ is the average value of the segmented object's gradient, reflecting the intensity of the vibration signal transformation. The larger the average gradient, the more intense the changes in the vibration signal. Let the gray image function of the segmented object be $f(x, y)$. Then the gradient is defined as

$$
G[f(x, y)]=\left[\begin{array}{l}
\frac{\partial f}{\partial x} \\
\frac{\partial f}{\partial y}
\end{array}\right] .
$$

In this paper, we take its length (also denoted as $G[f(x, y)]$ ) as the eigenvalue, which reflects the maximum rate of change of the segmented objects at point $(x, y)$, which is a scalar.

$$
G[f(x, y)]=\left[\left(\frac{\partial f}{\partial x}\right)^{2}+\left(\frac{\partial f}{\partial y}\right)^{2}\right]^{1 / 2}
$$

Energy spectrum entropy $\left(H_{V}\right)$

The spectrum entropy of the segmented objects is defined as the information entropy of the characteristic volume. Entropy is used to measure the average amount of information contained in a signal in information theory. The more ordered $a$ system is, the lower the information entropy is. In contrast, the more chaotic, disordered, and uncertain the system is, the higher the information entropy is. Therefore, the energy spectrum entropy was used to describe the confusion and uncertainty of the cylinder liner piston's severe vibration and characterize the vibration's complexity. The energy spectrum entropy is calculated as follows:

$$
H_{V}=-\sum_{i=1}^{n} P_{i} \log \left(P_{i}\right), P_{i}=\frac{V_{i}}{\sum_{i=1}^{n} V_{i}},
$$

where $V_{i}$ is the volume of the segmented object. 
Number of segmented objects $(N)$

The number of segmented objects reflects the frequency of the violent vibration in the whole signal period. The larger the number of segmented objects, the more vibration of the cylinder liner piston occurs. The number of segmented objects reflects the state of vibration and wear.

Discrete index of the shape of segmented object (e)

The shape dispersion index of a segmented object is the ratio of the boundary length to the area of the binary image corresponding to the largest feature volume, and reflects the dispersion and complexity of the vibration signal corresponding to the segmented object. The larger the discrete index is, the greater the dispersion and complexity of the vibration signal. The segmented object's boundary line is the transition from the binary image background to the target, which reflects the span of the vibration intensity from extensive to prominent. Therefore, the boundary value of the unit area (or unit energy) can reflect the vibration signal's dispersion and complexity. The discrete index $e$ of the characteristic volume is calculated as follows:

$$
\begin{gathered}
e=l^{2} / S \\
l=\sum_{k=1}^{N-1} \sqrt{\left(x_{k}-x_{k+1}\right)^{2}+\left(y_{k}-y_{k+1}\right)^{2}},
\end{gathered}
$$

where $\left(x_{k}, y_{k}\right)$ are the coordinates of the points on the segmented object's boundary and $N$ is the number of boundary points.

\subsection{Quantitative analysis of valve clearance}

According to the definition of the vibration signal characteristic parameters, the valve's characteristic parameters under four different clearance conditions were calculated. The results are shown in Table 2.

From the calculation results of the vibration characteristic parameters, it was found that the parameters $A_{m t}, E_{t}, P S D_{m}, E_{f}, G_{\text {mean }}, H_{V}$, and $N$ gradually increase as the working condition

Table 2

Calculation results of vibration characteristic parameters.

\begin{tabular}{lcccc}
\hline $\begin{array}{l}\text { Characteristic } \\
\text { parameter }\end{array}$ & $\begin{array}{c}\text { Working condition } \\
\text { of Val1 } \\
\text { Clearance: } 0.30 \mathrm{~mm}\end{array}$ & $\begin{array}{c}\text { Working condition } \\
\text { of Val2 } \\
\text { Clearance: } 0.35 \mathrm{~mm}\end{array}$ & $\begin{array}{c}\text { Working condition } \\
\text { of Val3 } \\
\text { Clearance: } 0.40 \mathrm{~mm}\end{array}$ & $\begin{array}{c}\text { Working condition } \\
\text { of Val4 }\end{array}$ \\
\hline$A_{m t}$ & 61.07 & 69.7 & 104.6 & 119.81 \\
\hline$E_{t}$ & 57.96 & 64.14 & 85.09 & 100.04 \\
\hline$P S D_{m}$ & 1.79 & 2.26 & 2.41 & 2.87 \\
\hline$E_{f}$ & 22.31 & 247.93 & 353.92 & 401.87 \\
\hline$G_{\text {mean }}$ & 1.53 & 1.76 & 1.64 & 2.21 \\
\hline$H_{V}$ & 0.26 & 0.17 & 0.31 & 0.6 \\
\hline$N$ & 19 & 20 & 26 & 664.35 \\
\hline$e$ & 583.58 & 294.31 & 316.6 & \\
\hline
\end{tabular}


changed from Vall to Val4. The results show that with increasing valve clearance, the energy of the valve-seat impact increases, and the maximum peak value (i.e., total vibration level) $A_{m t}$ increases with the valve clearance. With increasing total vibration energy, the time-domain second moment $E_{t}$ increases, the chaos and uncertainty of the vibration increase, and the spectrum entropy $H_{V}$ of the vibration characteristics increases.

To interpret the quantitative analysis/diagnosis of the diesel engines' valve clearance and quantify the fault information, it is necessary to establish a criterion function for obtaining the quantitative parameters of relevant faults. The established criterion function should be sensitive and reliable to reflect the degree of the fault. The specific steps of establishing the criterion function are as follows:

(1) The sample under the Vall working condition is taken as a reference sample. The selection of the reference sample defines the comparison standard of vibration characteristic parameters. In other words, the vibration signal corresponding to the valve clearance of $0.30 \mathrm{~mm}$ was selected as the reference sample.

(2) The sample's characteristic parameter values to be diagnosed or used in the simulation test were compared with the corresponding characteristic parameter values of the reference sample, and the dimensionless ratio was taken. The characteristic parameter values of each sample in the simulation test are shown in Table 3.

(3) The criterion function was obtained from a suitable weighting based on the dimensionless ratio of the reliable characteristic parameters that were sensitive to the fault. Because the maximum peak value $A_{m t}$ in the time domain, the second moment in the time domain $E_{t}$, the maximum peak value $P S D_{m}$ in the frequency domain, the average gradient of the segmented object $G_{\text {mean }}$, and the spectrum entropy $H_{V}$ of the segmented object are reliable parameters that are sensitive to changes in the gap, these five parameters were selected as the diagnosis basis. Because the time-domain second moment $E_{t}$ reflects the total vibration energy, the maximum peak value $P S D_{m}$ in the frequency domain will reflect the impact energy at a certain characteristic frequency. The comparison of the two parameters directly reflects the gap condition of the valve. Therefore, the weights of the two parameters should be larger, and the weights of the other parameters should be smaller. After several attempts, the weight of each parameter was finally selected, as shown in Table 4.

Table 3

Dimensionless characteristic parameters of vibration.

\begin{tabular}{lcccc}
\hline $\begin{array}{l}\text { Characteristic } \\
\text { parameter }\end{array}$ & $\begin{array}{c}\text { Working condition } \\
\text { of Val1 } \\
\text { Clearance: } 0.30 \mathrm{~mm}\end{array}$ & $\begin{array}{c}\text { Working condition } \\
\text { of Val2 } \\
\text { Clearance: } 0.35 \mathrm{~mm}\end{array}$ & $\begin{array}{c}\text { Working condition } \\
\text { of Val3 } \\
\text { Clearance: } 0.40 \mathrm{~mm}\end{array}$ & $\begin{array}{c}\text { Working condition } \\
\text { of Val4 }\end{array}$ \\
\hline$A_{m t} / A_{m t} 0.30$ & 1 & 1.14 & 1.71 & 1.96 \\
\hline$E_{t} / E_{t} 0.30$ & 1 & 1.11 & 1.47 & 1.73 \\
\hline$P S D_{m} / P S D_{m \_030}$ & 1 & 1.26 & 1.35 & 1.6 \\
\hline$E_{f} / E_{f \_} 0.30$ & 1 & 1.12 & 1.59 & 1.81 \\
\hline$G_{\text {mean }} / G_{\text {mean_0.30 }}$ & 1 & 1.15 & 1.07 & 1.44 \\
\hline$H_{V} / H_{V \_} 0.30$ & 1 & 0.65 & 1.19 & 2.31 \\
\hline$N / N_{0} 0.30$ & 1 & 1.05 & 1.37 & 1.74 \\
\hline$e / e \_0.30$ & 1 & 0.5 & 0.54 & 1.14 \\
\hline
\end{tabular}


Table 4

Weight table of vibration characteristic parameters.

\begin{tabular}{lccccc}
\hline Characteristic parameter & $A_{m t}$ & $E_{t}$ & $P S D_{m}$ & $G_{\text {mean }}$ & $H_{V}$ \\
\hline Weight coefficient $K$ & 0.2 & 0.3 & 0.3 & 0.1 & 0.1 \\
\hline
\end{tabular}

The decision function is defined as

$$
J=k_{1} \cdot \frac{A_{m t}}{A_{m t_{-} 0.30}}+k_{2} \cdot \frac{E_{t}}{E_{t_{-} 0.30}}+k_{3} \cdot \frac{P S D_{m}}{P S D_{m_{-} 0.30}}+k_{4} \cdot \frac{G_{\text {mean }}}{G_{\text {mean_ } 0.30}}+k_{5} \cdot \frac{H_{V}}{H_{V_{-} 0.30}},
$$

where $k_{1}, k_{2}, \ldots, k_{5}$ are the weight coefficients of the characteristic parameters of $A_{m t}, E_{t}, P S D_{m}$, $G_{\text {mean }}$, and $H_{V}$, respectively, and $A_{m t_{-} 0.30}, E_{t_{-} 0.30}, P S D_{\mathrm{m}_{-} 0.30}, G_{\text {mean } \_.30}$, and $H_{v_{-} 0.30}$ are the characteristic parameter values of Vall corresponding to the valve clearance of $0.30 \mathrm{~mm}$. The weight coefficient values in Table 4 and the characteristic parameter values of Vall in Table 2 are substituted into the decision function, which is simplified to obtain

$$
J=0.0033 A_{m t}+0.0052 E_{t}+0.1676 P S D_{m}+0.0654 G_{\text {mean }}+0.3846 H_{V} .
$$

The vibration characteristic parameters under the working conditions of Val1 to Val4 were substituted into the criterion function for calculation, and the corresponding criterion function values for different clearances were obtained as shown in Table 5 .

The results are shown in Fig. 9. According to the graph, the original data points can be fitted exponentially, as shown by the dotted line in Fig. 9. The relationship between the criterion function $J$ and the gap $\delta$ is obtained as follows:

$$
J=-5.6154+1.6684 \times e^{2.6036 \times \delta}+6.4834 \times e^{-2.6036 \times \delta} .
$$

Therefore, the one-to-one correspondence between the criterion function $J$ and the gap $\delta$ was established. Once the condition monitoring or fault diagnosis was carried out, the criterion function's value only need be calculated by the test method, and then the clearance value can be obtained from the one-to-one correspondence between the criterion function and the gap.

To test the accuracy of the quantitative analysis results, four inspection and test conditions were set on the diesel engine bench, as shown in Table 6 . Before the signal test of each group of working conditions, the diesel engine was run for about $45 \mathrm{~min}$, and the data were then collected after the diesel engine was running stably. For each group of working conditions, vibration signals were collected once every $10 \mathrm{~min}$, ten signals were collected in one group of working conditions, and 40 signals were collected from the four groups of working conditions. Five pieces of data were randomly selected from each group of working conditions, and a total of 20 pieces of signal data were extracted from four groups of working conditions. They were disordered but combined to form 20 test signals to be detected. 
Table 5

Criterion function values of different clearances.

\begin{tabular}{lcccc}
\hline \multirow{2}{*}{ Clearance $\delta$} & $\begin{array}{c}\text { Working condition } \\
\text { of Val1 }\end{array}$ & $\begin{array}{c}\text { Working condition } \\
\text { of Val2 }\end{array}$ & $\begin{array}{c}\text { Working condition } \\
\text { of Val3 }\end{array}$ & $\begin{array}{c}\text { Working condition } \\
\text { of Val4 }\end{array}$ \\
\cline { 2 - 5 } & $0.30 \mathrm{~mm}$ & $0.35 \mathrm{~mm}$ & $0.40 \mathrm{~mm}$ & $0.45 \mathrm{~mm}$ \\
\hline Criterion function value $J$ & 1.003 & 1.1228 & 1.418 & 1.7719 \\
\hline
\end{tabular}

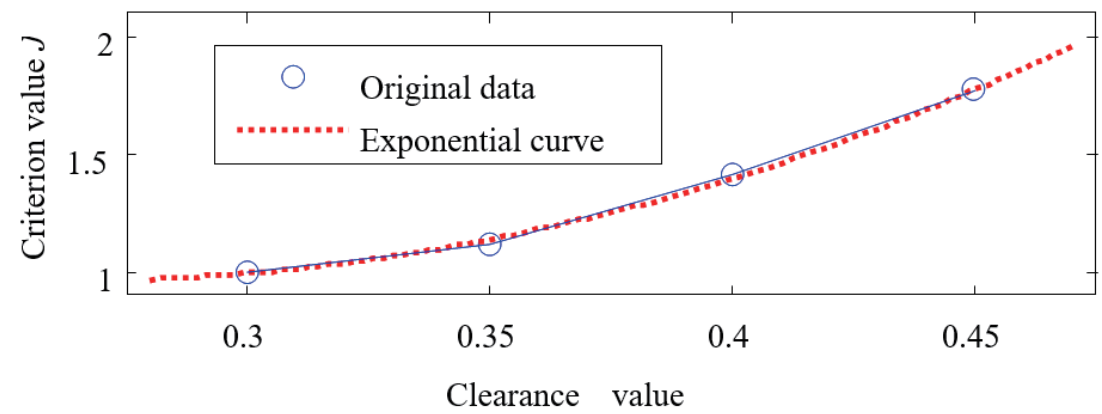

Fig. 9. (Color online) Diagram of the criterion function.

Table 6

Working conditions set for quantitative verification test of valve clearance.

\begin{tabular}{lcccc}
\hline Test No. & \multicolumn{4}{c}{ Working condition } \\
\cline { 2 - 5 } & $\begin{array}{c}\text { Exhaust valve } \\
\text { clearance }(\mathrm{mm})\end{array}$ & $\begin{array}{c}\text { Inlet valve } \\
\text { clearance }(\mathrm{mm})\end{array}$ & $\begin{array}{c}\text { Rotation speed } \\
(\mathrm{rpm})\end{array}$ & $\begin{array}{c}\text { Torque } \\
(\mathrm{N} \cdot \mathrm{m})\end{array}$ \\
\hline 1 & 0.35 & 0.3 & 1800 & 500 \\
\hline 2 & 0.40 & 0.3 & 1800 & 500 \\
\hline 3 & 0.45 & 0.3 & 1800 & 500 \\
\hline
\end{tabular}

The 20 test signals to be detected were analyzed by the gap quantitative analysis method, and their gap values were calculated. To measure the accuracy of the calculation results, the error between the measured value and the real value was set to be within $0.03 \mathrm{~mm}$ (accounting for $10 \%$ of the reference gap value). It is considered that the detection result was accurate, and the status recognition and diagnosis were successful. Otherwise, it was considered that an error occurred and the recognition failed. The test results are shown in Fig. 10. The number of successes and the success rate of the inspection results are shown in Table 7. The total success rate is the ratio of the number of test groups successfully identifying the gap to the total number of groups in all four tests and was $90 \%$. The proposed method can thus be used for condition monitoring and fault diagnosis in the quantitative identification of valve clearance.

\subsection{Comparison with other methods for analysis of engine vibration}

Previous studies on engine vibration signals mainly analyzed the spectral characteristics of the vibration. Moreover, the extracted characteristic parameters were mainly statistical 


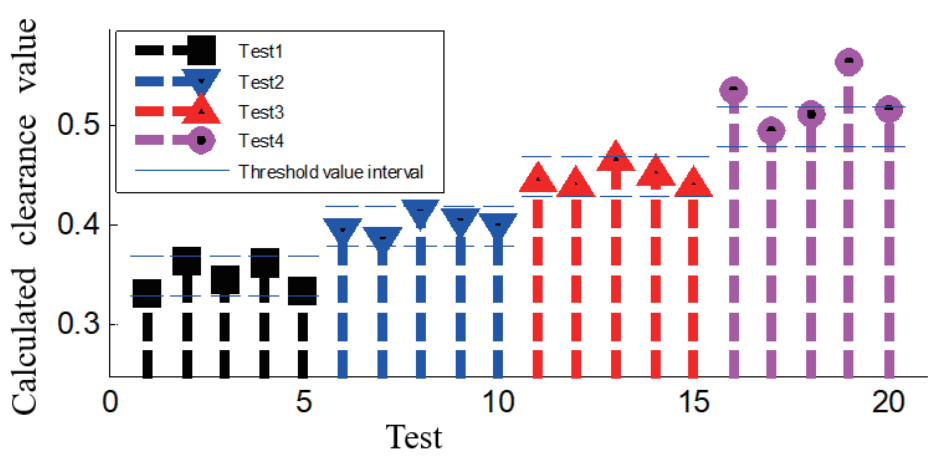

Fig. 10. (Color online) Distribution of test signal detection results.

Table 7

Detection under four states.

\begin{tabular}{lrrrr}
\hline & test 1 & test 2 & test 3 & test 4 \\
\hline Number of successes & 5 & 5 & 5 & 3 \\
\hline Number of failures & 0 & 0 & 0 & 2 \\
\hline Success rate $(\%)$ & 100 & 100 & 100 & 60 \\
\hline Total success rate $(\%)$ & \multicolumn{5}{c}{90} \\
\hline
\end{tabular}

Table 8

Comparison with other methods for the analysis of engine vibration.

\begin{tabular}{lccccc}
\hline Methods & $\begin{array}{c}\text { Time-domain } \\
\text { information }\end{array}$ & $\begin{array}{c}\text { Frequency } \\
\text {-domain } \\
\text { information }\end{array}$ & $\begin{array}{c}\text { Spatial } \\
\text { characteristics }\end{array}$ & $\begin{array}{c}\text { Quantitative } \\
\text { analysis }\end{array}$ & $\begin{array}{c}\text { Nonlinear and } \\
\text { non-stationary } \\
\text { analysis }\end{array}$ \\
\hline $\begin{array}{l}\text { Power spectrum } \\
\text { analysis }\end{array}$ (3,4) & $\mathrm{N}$ & $\mathrm{Y}$ & $\mathrm{N}$ & $\mathrm{Y}$ & $\mathrm{N}$ \\
\hline $\mathrm{ICA}^{(8,9)}$ & $\mathrm{Y}$ & $\mathrm{N}$ & $\mathrm{N}$ & $\mathrm{N}$ & $\mathrm{N}$ \\
\hline $\begin{array}{l}\text { Wavelet packet } \\
\text { analysis }\end{array}$ (5-7) & $\mathrm{Y}$ & $\mathrm{Y}$ & $\mathrm{N}$ & $\mathrm{Y}$ & $\mathrm{N}$ \\
\hline Proposed method & $\mathrm{Y}$ & $\mathrm{Y}$ & $\mathrm{Y}$ & $\mathrm{Y}$ & $\mathrm{Y}$ \\
\hline
\end{tabular}

parameters based on the time and frequency domains. However, it was difficult to assess the extent of wear and the engine motion mechanism's health using these parameters. Table 8 shows a comparison of the information obtained from the method in this study and other methods. The method proposed in this study provides information in the time and frequency domains. As pointed out earlier in this paper, power spectrum analysis cannot obtain time-domain information, and it is difficult to confirm the number of independent components in ICA and amplitude information is lost, making it impossible to use for quantitative analysis. Nonlinear and nonstationary analyses are enabled using our proposed method.

\section{Conclusion}

In this paper, a local wave analysis method was applied to the nonstationary vibration response analysis of a diesel engine surface, and the impact response of the valve seat was 
analyzed. The vibration characteristics were extracted by CWT secondary image analysis. The internal relationship between different valve clearance and valve-seat impact vibration characteristics was revealed, and methods of valve clearance monitoring and abnormality diagnosis were also established.

(1) Based on the local wave decomposition and reconstruction of abnormal vibration signals from the diesel engine gap, the signals' energy distribution characteristics under different clearance conditions showed obvious differences in the time and frequency domains. The maximum peak value $A_{m t}$ in the time domain, the maximum peak value $P S D_{m}$ in the frequency domain, the second moment in the time domain $E_{t}$, and the total energy $E_{f}$ in the frequency domain were extracted to investigate the time-frequency characteristics of the abnormal vibration signal of a diesel engine. The characteristics of the power spectrum signal provide a reliable foundation for recognizing the working state of diesel engines.

(2) The CWT time spectrum secondary image and image segmentation technology were used to extract the characteristic parameters of vibration signals, such as the average gradient $G_{\text {mean }}$, quantity $N$, discrete exponent $e$, and energy spectrum entropy $H_{V}$. These were used to characterize the diesel engine gap abnormal vibration signals and realize the quantitative characterization of abnormal vibration signals' characteristics.

(3) On the basis of local wave decomposition and reconstruction and CWT technology, the vibration characteristic parameters describing the diesel engine clearance state were extracted, and the valve clearance state identification and diagnosis criterion function were then established, enabling the quantitative monitoring of the valve clearance and fault diagnosis of abnormal valve clearance.

\section{Acknowledgments}

This work was supported by the Fujian Provincial Department of Ocean and Fisheries (No. FJHJF-L-2020-6), the Fund of Fujian Province Key Laboratory of Ship and Ocean Engineering and the Ministry of Science and Technology, Taiwan (MOST 108-2221-E-158-003 and MOST 109-2622-E-035-022).

\section{References}

1 L. Liu, G. M. Zhang, and C. Zhang: Internal Combust. Engine Powerplant 36 (2019) 80. http://www.cnki.com. cn/Article/CJFDTOTAL-SDNR201904015.htm

2 Y. Liu, J. H. Zhang, and F. R. Bi: Meas. Sci. Technol. 26 (2015) 025003. https://doi.org/10.1088/0957$\underline{0233 / 26 / 2 / 025003}$

3 P. Forsberg, P. Hollman, and S. Jacobson: Wear 271 (2011) 2477. https://doi.org/10.1016/j.wear.2010.11.039

4 K. Jafarian, M. Mobin, R. Marandi, and E. Rabiei: Measurement 128 (2018) 527. https://doi.org/10.1016/j. measurement.2018.04.062

5 G. M. Szymański and F. Tomaszewski: Mech. Syst. Sig. Process. 68 (2016) 479. https://doi.org/10.1016/j. ymssp.2015.07.015

6 S. Vulli, J. F. Dunne, R. Potenza, and D. Richardson: J. Sound Vib. 321 (2009) 1129. https://doi.org/10.1016/j. jsv.2008.10.011

7 H. B. Zheng, Z. Y. Li, and X. Z. Chen: Sound Vib. 20 (2002) 267. https://doi.org/10.16236/j.cnki. nrjxb.2002.03.016

8 J. Lin, and L. Qu: Trans. CSICE 234 (2000) 135. https://doi.org/10.1006/jsvi.2000.2864 
9 Y. P. Cai, A. H. Li, and Y. P. He: Proc. 2010 2nd Int. Conf. Advanced Computer Control (2010) 27-29. https:// doi.org/10.1109/ICACC.2010.5487142

10 W. Deng, S. J. Zhang, and H. M. Zhao: IEEE Access 6 (2018) 35024. https://doi.org/10.1109/ ACCESS.2018.2834540

11 Y. B. Jing, C. W. Liu, and F. R. Bi: Chin. J. Mech. Eng. 30 (2017) 991. https://doi.org/10.1007/s10033-017-0140-2

12 C. M. Huang, P. Zhang, and H. L. Yu: Appl. Mech. Mater. 239-240 (2013) 430. https://doi.org/10.4028/www. scientific.net/AMM.239-240.430

13 X. Wang, C. W. Liu, and F. R. Bi: Mech. Syst. Signal Process. 41 (2013) 581. https://doi.org/10.1016/j. ymssp.2013.07.009

14 X. Y. Bi, S. Q. Cao, and D. M. Zhang: Energies 12 (2019) 661. https://doi.org/10.3390/en12040661

15 Q. C. Gao and F. C. Hu: J. Wuhan Univ. Technol. (Transportation Science \& Engineering) 4 (2019) 746. http:// www.cnki.com.cn/Article/CJFDTOTAL-JTKJ201904033.htm

16 M. He, Y. Zhou, Y. Li, G. Wu, and G. Tang: Measurement 156 (2020) 107582. https://doi.org/10.1016/j. measurement.2020.107582

17 L. Xu, S. Chatterton, and P. Pennacchi: Mech. Syst. Sig. Process. 148 (2021) 107174. https://doi.org/10.1016/j. ymssp.2020.107174

18 Y. Liu, J. H. Zhang, and L. Ma: Neurocomputing 177 (2016) 600. https://doi.org/10.1016/j.neucom.2015.11.074

19 Z. Li, X. C. Chen, and Z. B. Liu: Trans. CSICE 3 (2001) 241. https://doi.org/10.16236/j.cnki.nrjxb.2001.03.011

20 H. Y. Zhang, C. Y. Jia, and B. Yu: Syst. Eng. Electron. 27 (2005) 632. http://www.cqvip.com/ qk/95985x/200504/15566006.html

21 C. Gao, and J. G. Sun: J. Jilin Univ. (Earth Science Edition) 5 (2015) 1523. https://doi.org/10.13278/j.cnki. jjuese. 201505301

\section{About the Authors}

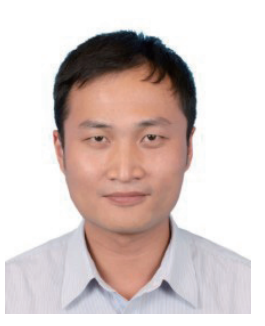

Chaoming Huang received his Ph.D. degree in 2011 from the College of Marine Engineering, Dalian Maritime University (DMLU). He is now a lecturer at DLMU. His major research interests include vibration and noise control, friction and wear control, and fault diagnosis and prediction.

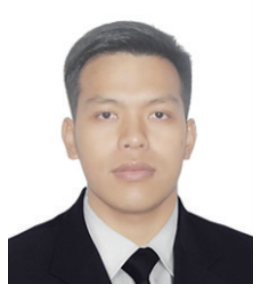

Jie Li has been pursuing a bachelor's degree in the College of Marine Engineering, Dalian Maritime University since 2019. His major research interests include vibration and noise control and vibration feature extraction.

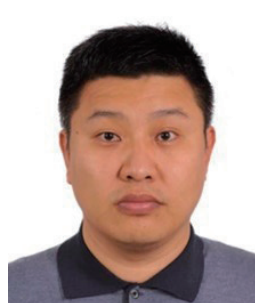

Xin Wang started his career at the Information and Communication Office of China Coast Guard after obtaining a bachelor's degree from the College of Maritime Engineering, Dalian Maritime University. His major research interests include vibration and noise control. 


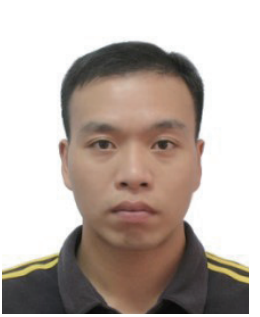

Jianbin Liao is an associate professor of Jimei University. He is currently a Ph.D. candidate of Dalian Maritime University. He received his B.S. and M.S. degrees in marine engineering from Jimei University in 1998 and 2007, respectively. His current research interests include marine engineering and fault diagnosis.

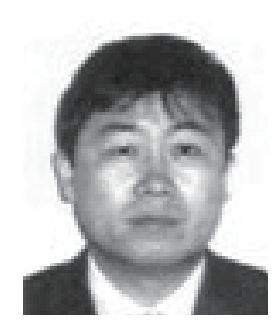

Hongliang Yu received his Ph.D. degree in 2002 from Kobe University of Mercantile Marine, Japan. He is now a professor at Jimei University, Xiamen, China. His major research interests include modern marine engineering management, vibration fault diagnosis, module shipbuilding, and reliability.

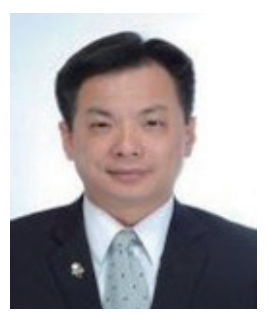

Chih-Cheng Chen has been a professor at Jimei University, China, since 2017. He became a member of IEEE in 2011 and a senior member in 2016. He earned his M.S. and Ph.D. degrees from the Department of Mechatronics Engineering, National Changhua University of Education. His research interests include AIoT technology and RFID applications.

(201761000018@jmu.edu.cn)

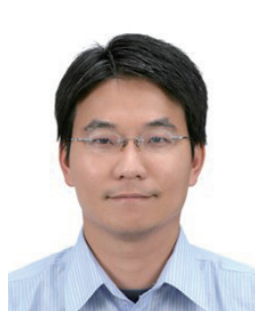

Kun-Ching Wang is a professor of mechanical and computer-aided engineering at Feng Chia University in Taiwan. He is also currently serving as the deputy secretary general of the Taiwan Association of Systems Science and Engineering (TASSE). His research interests include speech/audio processing and recognition, machine learning, computer vision, advanced driver assistance systems, and IoT applications. His research results have been published in over 40 journal and conference papers. 